\section{SILICON TECHNOLOGY FOR THE SEMICONDUCTOR INDUSTRY}

\section{Silicon Semiconductor Technology}

By W. R. Runyan. (Texas Instruments Electronics Series.) Pp. ix +277. (New York: MeGraw-Hill Book Company, Inc.; Maidenhead: McGraw-Hill Publishing Company, Ltd., 1965.) 132s.

ERMANIUM and silicon have dominated semiconductor technology for the past twenty years and the progress made with their preparation has maintained for them leading places in semiconductor physics. The spur for the technological developments was not, however, primarily one of expectations of discoveries of new physical effects but rather the prospects of the large-scale manufacture of junction transistors. Germanium received most attention at first; that rare element, previously little investigated, was soon to be made available more nearly completely purified and with greater crystalline perfection than any other substance. Its purification owed much to the development of zone refining, while the production of the large monocrystals with the controlled levels of impurity content required by the makers of devices was made possible only by the greatly improved control of the Czochralski method of pulling crystals from the melt and the adaptation of zone refining to zone levelling. No book has integrated these developments, other than by collecting together many of the original papers.

Similar studies with silicon started a little later; here again the spur was the prospect of industrial exploitation. Compared with germanium, silicon is a very abundant element and its supply does not depend on residues from other industries. Its conductivity, too, can be made $n$ - or $p$-type, but its energy gap is one and a half times as great. For a given donor or acceptor concentration, it therefore retains its extrinsic properties to higher temperatures, and junction devices made in it can be used at temperatures up to $150^{\circ} \mathrm{C}$ or higher. The oxide which can easily be grown on it is not hygroseopic and might be expected to protect junctions from the detrimental effects of water vapour which were so noticeable with early germanium devices.

Progress with silicon benefited from that with germanium, but was not spectacular at first; the higher melting point and greater chemical reactivity presented problems. None proved insuperable, however, and the advantages predicted began to be realized. The silicon devices now available include high-voltage rectifiers, thyristors handling currents of $100 \mathrm{amp}$ or more, transistors with gainbandwidth products of $1,000 \mathrm{Mc} / \mathrm{s}$, varactor diodes, solar cells and fully integrated electronic circuits; they testify to the success of the basio technology of this element. But, once again, no author had devoted a book to silicon technology until this recent addition to a newly launched series.

Silicon Semiconductor Technology is an educational book as well as one of reference. The order of presentation might be questioned, but frequent users will have little complaint to make on that score. After a short historical note, the second and third chapters describe briefly the chemical purification of silicon and methods for casting it into several shapes. The fourth chapter is more detailed in its account of crystal growing. It concentrates on pulling from the melt and growth from the vapour phase on to a substrate. The segregation of impurities during pulling or zone melting is well looked after, with data for several important olements. The next chapter surveys crystal systems before examining the diamond lattice in particular and telling how to determine the orientation of silicon crystals and the presence of twinning and of grain boundaries; but it fails to link up with the defects of the crystals grown by the methods of the previous chapter. The sixth chapter returns to doping procedures, listing the behaviour of the elements in common use as donors and acceptors, without adding much quantitative information to that already given. Diffusion of dopants is the subject of the next chapter; here the author keeps device technology to the fore with his choice of geometrical conditions and detailed attention to boron and phosphorus, the two elements of which the diffusion must be so accurately controlled in the planar technology. The next three chapters describe very competently the electronic, optical and other physical properties of monocrystalline silicon, relating them to composition. They would not be out of place in a book on semiconductor physics, but are just as needed here, for so many physical properties have to be taken account of in device technology. The final chapter switches to metallurgy, in the form of diagrams of binary phase systems, nearly forty in all, with silicon as one of the two elements throughout.

The author pleads that any description of surface behaviour would demand far more space than he could spare; but some account of the technology of thermally grown layers of silicon dioxide, and their role, was surely justified. Room for a chapter could have been found by omitting some absorption spectra and the phase diagrams of silicon and many metals with which it is only rarely associated, and by condensing some other figures.

The book is excellently produced, and almost all the basic technological data vital to the silicon semiconductor industry are presented clearly and in sufficient detail. Although the writing offends occasionally, particularly by its frequent and sometimes indiscriminate use of 'This' and 'this', the book is commended to everyone with a serious interest in the subject.

J. R. Trllman

\section{SOLAR RADIO ASTRONOMY}

\section{Solar Radio Astronomy}

By Mukul R. Kundu. Pp. $x i+660$. (New York and London: Interscience Publishers, a Division of John Wiley and Sons, 1965.) $150 \mathrm{~s}$.

RADIO astronomy has taken a vigorous place in modern research since the Second World War. For ten years it was a compact subject, not too large to be covered by one reasonably comprehensive text. By 1956 Shklovsky recognized that this could not continue, and his book of that year dealt only with radio objects beyond the solar system. Now Dr. Kundu has shown that it requires a substantial book to cover the solar part of radio astronomy. It is very satisfying that, at the present time, the solar part is clearly defined and there is very little ambiguity concerning the material that should be included in a book entitled Solar Radio Astronomy.

The introductory chapters, 1-4, cover the relevant optical observations, radio propagation and emission, and observing techniques. The radio propagation theory is not very complete and reference to other texts may be necessary. Three radio emission processes are discussed under the titles "Bremsstrahlung", "Gyro-synchrotron Radiation", and "Cerenkov Radiation". These sections give the essentials of thermal, synchrotron and plasma emissions, all of which are required for explaining solar phenomena. There is a useful discussion on the Ginzburg and Zheleznyakov theory of coupling between plasma and electromagnetic waves. The developments of interferometry for solar radio purposes are described.

The central theme of the book is contained in Chapters 5-12, which deal systematically with the emission described as: quiet sun; slowly varying emission; cm bursts; $\mathrm{dm}$ bursts; meter bursts of types II, III, $U$ and V; type IV radiation; noise storms and type I bursts. Each type of radiation is treated in a separate chapter and this makes it somewhat difficult to interrelate the burst phenomena until the threads are gathered together in Chapter 11. The great significance of the type IV synchrotron emission and its various manifestations is well portrayed. 